\section{How antidepressants work}

\author{
New perspectives on the pathophysiology \\ of depressive disorder
}

IAN C. REID and CAROLINE A. STEWART
Antidepressant therapy, like many treatments in psychiatry, is an empirical affair. Although there is little doubt that chemical antidepressants work, modern drugs are no more efficacious, and act no more rapidly, than the agents discovered by chance more than four decades ago. Progress in the neuropharmacology of depressive disorder has provided us with an ever more fine-grained picture of the activities of monoamine systems and their modulators, rather than a conceptually more sophisticated view of brain function in affective illness. The limitations of the monoamine hypothesis are well recognised: indeed, the fact that monoaminergic modulators are effective in the treatment of depressive disorder need not implicate monoamines in the aetiology of the illness at all. It has long been suspected that changes in neural factors 'downstream' from the effects of monoamine modulation will prove to be crucial elements in understanding the biology that underlies depression.

\section{AN 'IDEAL' NEUROBIOLOGYOF DEPRESSIVE DISORDER}

A comprehensive neurobiology of the aetiology of depressive disorder could never be served exclusively by a monoaminergic hypothesis, no matter how detailed or modified. This is not to dismiss the importance of monoamines - simply a recognition that an 'ideal' aetiological model must take account of events beyond the explanatory scope of monoamine neuroreceptor regulation alone. These include genomic/ molecular activities, cellular changes, the properties of networks of neurons and the characteristics of discrete brain structures or modules and their neuropsychological correlates. Any comprehensive neurobiology of depressive disorder must accommodate in a plausible way such factors as early experience, stress and the impact of social interaction. The model would also have to account for the effectiveness of monoamine modulation, of course, in addition to the other physiological and psychological characteristics of depressive disorder, including, for example, the role of corticosteroid abnormalities, neuroanatomical changes and cognitive dysfunction.

Here, we attempt to draw together a range of recent neurobiological studies in order to construct a preliminary framework for an enriched neurobiology of depressive disorder.

\section{MOLECULAR AND CELLULAR FINDINGS: THE NEUROTROPHIC PERSPECTIVE}

Ronald Duman and his colleagues at Yale University have reported some of the most exciting new findings relating to antidepressant action at the cellular/molecular level. Studying the effects of chronic antidepressant therapy on cellular function in the rat brain, they have established that treatments from different antidepressant classes appear to have the common property of increasing the expression of neuroprotective proteins, important in the function and growth of neurons (for a review see Duman et al, 1997). This effect may represent the final common pathway of a complex chain of intracellular events triggered by antidepressant treatments that, none the less, are chemically very different. Antidepressant-induced increases in presynaptic monoamine release arise via a variety of mechanisms (monoamine oxidase inhibition, reuptake blockade, presynaptic or somatodendritic autoreceptor downregulation), and result in activation of a range of post-synaptic receptors that are coupled to 'second messenger' signal transduction mechanisms. Activation of these enzyme systems ultimately results in the phosphorylation of transcription factors that control gene expression. Duman and his co-workers have focused on the activities of the transcription factor 'cAMP response-element binding protein' (CREB). Levels of messenger RNA (mRNA) coding for this protein, and the protein itself, are increased in the hippocampus following chronic antidepressant administration over a period that mirrors the delayed response observed clinically during antidepressant treatment. The Yale group propose that 
CREB activates genes controlling the expression of the neurotrophic protein designated 'brain-derived neurotrophic factor' (BDNF) and its receptor, tropomyosin receptor- related kinase $\mathrm{B}$ (TrkB). In accord with this hypothesis, they have shown parallel increases in BDNF and TrkB mRNA in the hippocampus of rats exposed chronically to a wide range of antidepressants.

These are exciting observations for a number of reasons. First, BDNF belongs to a family of growth factors that control a variety of important neural activities ranging from cell differentiation during brain development to cell survival in the mature brain. Second, rats exposed to restraint stress show a reduction in BDNF expression in the hippocampus, and this effect is opposed by antidepressants (Smith et al, 1995). Third, direct infusion of BDNF itself into rat brain has putative antidepressant effects in preclinical animal models of depression (Siuciak et al, 1997). Last, but not least, these findings indicate a range of novel molecular targets for the development of new antidepressant therapies.

The fact that stress can down-regulate the expression of a protein responsible for the maintenance of cellular viability might relate to mechanisms that support adaptive stress responses, rather than harmful stress effects. However, the finding is consistent with the observation that a number of brain structures, including the hippocampus, may become atrophic in depressive disorder (e.g. Shah et al, 1998). It has been suggested that depression may represent a subtle neurodegenerative disorder, and that the antidepressant regulation of neuroprotective factors like BDNF acts to reverse such effects (Altar, 1999). Even electroconvulsive stimulation, so long viewed as potentially damaging to neurons, promotes BDNF expression and induces sprouting of hippocampal neurons (Vaidya et al, 1999).

These findings assume special significance now that it is apparent that new neurons are generated throughout life in the hippocampus of a variety of species, including humans. It has been suggested that a spectrum of factors threatens the balance of neural viability in the hippocampus, ranging from genetic influences, through the effects of stress and elevated corticosteroid levels, to sundry insults such as ischaemia, hypoglycaemia, neurotoxins and viral infections (Duman et al, 1997).
It is important to recognise that hippocampal cell viability may be sensitive to relatively subtle psychological events. Brief social stressors, for example, have been shown recently to interfere with cell proliferation in the dentate gyrus of the hippocampus of primates (Gould et al, 1998). In this study, adult marmosets that had been housed individually were transferred to the home cage of another, unfamiliar marmoset for 1 hour. This enforced intrusion on the territory of another monkey is very stressful, and though the 'intruder' monkeys remained protected within the arena by a smaller cage, they adopted submissive postures and showed signs of distress and autonomic arousal. The intruder monkeys were then injected with a thymidine analogue, which is incorporated into proliferating cells, acting as a marker for neurogenesis. Immunohistochemical analysis showed subsequently that the stressor had reduced the rate of cell proliferation to less than two-thirds of the proliferation rate observed in the hippocampi of control monkeys. Antidepressant treatments may also prove protective in these circumstances: Madsen et al (2000) have recently shown that repeated electroconvulsive stimulation (ECS) in rats almost doubles the number of new-born cells observed in the dentate gyrus of the hippocampus compared with controls. Indeed, it is conceivable that this bolstering of neuronal survival may turn out to be a useful property of antidepressant treatments beyond depressive disorder. Potential applications include post-traumatic stress disorder, and more clearly neurodegenerative diseases, such as Alzheimer's and Parkinson's disease.

\section{PHYSIOLOGY: NETWORKS OF NEURONS}

The expression of BDNF does more, however, than simply determine whether neurons live or die: it also regulates the way in which networks of neurons communicate with one another. In particular, it influences the activity of a subclass of excitatory amino acid receptor, the $\mathrm{N}$-methylD-aspartate (NMDA) receptor (Levine et al, 1998), that, in turn, determines changes in neural connection strength by regulating important forms of synaptic plasticity such as long-term potentiation (LTP) and longterm depression (LTD). The induction of LTP represents an increase in synaptic connectivity, whereas LTD represents a decrease in synaptic connectivity. This ability to change patterns of neural connection strength rapidly, and in a lasting way, can be demonstrated throughout the mammalian forebrain. In the hippocampus, for example, it is highly probable that changes in patterns of connectivity determine aspects of memory function and responses to stress, while in the amygdala, similar plastic phenomena play an important role in fear responses (for a review see LeDoux, 1996).

These technical and conceptual advances permit us to begin to ask questions about the dynamics of information processing in the brain such that neuropsychology and neurophysiology are drawn together in a way that traditional neurochemical analysis alone could never achieve. Recognising, for example, that stressors may change patterns of connection strength in neural networks begins to make tractable the analysis of the nature of the information processing (and its dysfunction) that might underlie the cognitive and emotional changes that accompany affective disorder. It is this potential which most sharply differentiates the perspective elaborated here from neuroreceptor accounts of the neurobiology of depressive disorder. Neural network models of psychopathology are already beginning to emerge (e.g. Jeffery \& Reid, 1997).

The effects of antidepressant agents on BDNF and excitatory amino acid function described above may explain previous demonstrations of antidepressant-induced modulation of synaptic connectivity (e.g. Stewart \& Reid, 1993). We have shown in several in vivo studies that repeated (but not single) ECS consistently enhances synaptic connectivity in the dentate gyrus of the rodent (Stewart \& Reid, 1993; Reid \& Stewart, 1997). These effects lasted for at least $\mathbf{4 0}$ days after the end of the course of ECS, and developed incrementally seizure by seizure during the course of stimulation. The maximum effect was reached only after four to six seizure applications, each spaced by 48 hours. These findings are consistent with clinical observations relating to the efficacy of electroconvulsive therapy (ECT) in humans. Prior administration of the NMDAreceptor-associated channel-blocker ketamine prevented the change in connectivity, implicating excitatory amino acid neurotransmission in the actions of ECS (reviewed by Petrie et al, 2000). 
Given the important role that LTP may play in memory formation, the modification of plasticity observed might account for the amnesic effects of seizure activity (Reid \& Stewart, 1997). However, we have recently reported that chronic administration of fluoxetine, which does not share the amnesic properties of ECS, induced similar up-regulation of connectivity in the dentate gyrus (Stewart \& Reid, 2000). This does not exclude the interesting possibility that effects of ECS on synaptic plasticity in other subfields of the hippocampus, or in other brain structures, are responsible for the transient anterograde amnesia that follows seizure activity in humans and other species. This would imply that fluoxetine and ECT have differential effects on synaptic plasticity in different brain areas.

It seems likely that there are multiple routes to the changes in connectivity induced by different antidepressants. There is evidence that chemical antidepressants may interact directly with the excitatory amino acid systems that underpin changes in synaptic connection strength, in addition to enhancing BDNF expression. A number of different antidepressant agents have regulatory actions at the NMDA receptor complex and some NMDA receptor antagonists themselves have antidepressant properties in animal models that predict the clinical effectiveness of traditional drugs (Skolnick, 1999). These findings suggest that it is the ability to modify synaptic plasticity that is the crucial feature of clinically effective antidepressants, rather than the enhancement of neuronal survival alone (Petrie et al, 2000).

\section{STRESS AND SYNAPTIC PLASTICITY}

How might antidepressant-induced changes in the molecular and physiological events that underpin the regulation of synaptic connectivity relate to the aetiology of depressive disorder? As noted above, stressors down-regulate BDNF expression, increasing neuronal vulnerability and reducing cell proliferation in the hippocampus. Stress has a parallel impact on synaptic plasticity. Early studies showed that exposure to inescapable stressors reduced markedly the degree to which LTP could be induced in slices of hippocampus isolated in vitro from rats. The most potent stressors in this regard are those known to induce 'learned helplessness', a well-known behavioural model of depressive disorder (for a review see Kim \& Yoon, 1998).

Like humans, individual rats vary in their response to a given stressor. Indeed, variations in stress-induced excitatory synaptic changes correlate with variations in recognised somatic manifestations of stress, such as stomach ulcer formation. Henke (1990) has shown that stress is more likely to promote ulcer formation in the stomachs of rats that show a down-regulation of connectivity in the dentate gyrus of the hippocampus in response to restraint stress. Conversely, he observed that stress-resistant rats, which did not develop ulcers during the procedure, showed an increase in excitatory post-synaptic potential in the dentate cell fields. Furthermore, Henke was able to reduce ulcer formation in stressed rats by artificially inducing LTP via electrical stimulation of the afferent pathways to the dentate gyrus (Henke, 1989). This stressprotective change in electrophysiological signature produced by direct manipulation of hippocampal activity is consistent with the changes we have observed following chronic antidepressant administration and repeated electroconvulsive stimulation in rats (Stewart \& Reid, 2000).

Studies that are more recent indicate that stressors may alter the dynamic balance between increases and decreases in synaptic connectivity such that LTD is favoured. Kim \& Yoon (1998) have suggested that a complex relationship between past and current synaptic activity exists, which controls the plastic properties of neural networks (so-called 'metaplasticity' - a changing capacity for plasticity). They highlight the importance of intracellular calcium levels, regulated by excitatory amino acid receptors, in determining whether LTD (low calcium levels), LTP (high calcium levels) or excito-toxic vulnerability (very high calcium levels) occurs. Again, a continuum from cellular dysfunction to cell death is proposed, determined by the 'history' of neural activity, which is in turn modulated by experience.

\section{ROLE OF GLUCOCORTICOIDS}

Internal hormonal responses also control the vulnerability of plastic systems to stress. Glucocorticoids, released during stress and often abnormally regulated in depressive disorder, have important effects on the hippocampus and its plastic mechanisms. The hippocampus is well endowed with adrenal steroid receptors - high-affinity type I (mineralocorticoid) receptors and low-affinity type II (glucocorticoid) receptors. Differential activation of these receptor subtypes has been shown to upregulate or down-regulate synaptic connectivity, depending on stress hormone levels (Pavlides et al, 1995). High levels of corticosterone (the principal rodent stress hormone) blocked the induction of LTP and facilitated the induction of LTD in rats and thus down-regulated connectivity. On the other hand, lower levels of corticosterone (favouring type I receptor activation over the activation of the loweraffinity type II receptors) up-regulated connectivity by facilitating LTP. Selective glucocorticoid (type II) receptor agonists mimic the effects of high corticosterone levels, whereas type II antagonists have been shown to prevent stress-induced changes in synaptic plasticity (Xu et al, 1998). Type II glucocorticoid receptor antagonists are being evaluated currently as candidate antidepressant agents. Stress hormone effects may be linked to BDNF activity, as high levels of corticosterone have been found to down-regulate BDNF mRNA expres-sion and BDNF protein content in the hippocampus (Schaaf et al, 1998).

Clearly, there is the potential for a dynamic interaction between stress, corticosterone, BDNF and synaptic plasticity modified by both the genetically determined integrity of the hippocampus, and its past history of psychological and physiological insults.

\section{EARLY ADVERSE EXPERIENCE AND HIPPOCAMPAL FUNCTION}

The history of hippocampal insult may extend back to very early experiences indeed. Disruption of early postnatal motherinfant relationships in rats has lasting effects on hippocampal physiology. In a series of studies, Bronzino and colleagues (Kehoe et $a l, 1995)$ have described enhanced LTP induction and duration in the dentate gyrus of rats that had experienced repeated episodes of brief separation from their mothers and siblings. The rats had been isolated for 1 hour each day on eight occasions, between their 2nd and 9th days of life. Almost 3 weeks later, changes in the plasticity of the hippocampal system were 
evident, with greater effects in male than female rats.

Studies of the relationships between stressful experience and the plasticity of neural systems are contributing to an emerging biology of the impact of early experience and later adverse life events on limbic neural systems. A coherent account of the biology of early adversity and stressful life events (well-established risk factors in depressive disorder) will be crucial to any future satisfactory neurobiology of human affective disorder. The mechanisms of antidepressant action reviewed above clearly have the potential to interact with the pathoplastic effects of adverse experience described here.

\section{BRAIN MODULES: STRUCTURE AND NEUROPSYCHOLOGICAL FUNCTION IN DEPRESSION}

Much of the research discussed so far has focused on the hippocampus. Clearly, however, the hippocampus is not the only brain structure involved in depressive disorder, nor are the mechanisms described above exclusive to the hippocampal formation. Dynamic changes in synaptic connectivity such as LTP can be demonstrated in slices of rat brain taken from medial frontal cortical areas (Vickery et al, 1997), implicated in depressive disorder in humans in a number of imaging studies. Drevets et al (1997), for example, have described both structural and functional abnormalities in the anterior cingulate and associated prefrontal cortex. They report both decreased activity and a reduction in cortical volume of the left subgenual prefrontal cortex in patients with depression using magnetic resonance imaging and positron emission tomography. To an extent, therefore, the hippocampal neurobiology described here may serve as a model system for understanding the physiology of other brain areas in depressive disorder. However, varying neural arrangements in diverse brain structures will possess different information processing properties, contributing to distinct neuropsychological functions. In this way, the pathological effects of stress on synaptic connectivity will be expressed differently depending on the structures involved. Indeed, it is conceivable that neuropsychologically distinct subgroups of depressive disorder may eventually be defined.

\section{HIPPOCAMPUS AND DEPRESSIVE DISORDER}

The hippocampus is also specifically implicated in depressive disorder. Numerous studies have confirmed the presence of memory dysfunction in patients with depression. Recently, Shah et al (1998) have demonstrated a correlation between hippocampal atrophy and impaired verbal learning in patients with chronic, treatmentresistant depression. Furthermore, the neuropsychological hallmark of hippocampal dysfunction - a dissociation between memory for events (impaired explicit memory) and memory for skills and procedures (preserved implicit memory) - is encountered in patients with depression (Bazin et al, 1994). Usually, anterograde learning impairments observed in subjects with depression are less striking clinically than those encountered in patients with amnesia, but formal evaluation reveals marked deficits. Abas et al (1990), for example, found that the performance of elderly patients with depression on a delayed match-to-sample task (a task sensitive to hippocampal dysfunction) was as poor as in patients with Alzheimer's disease. Although performance improved following recovery from depression, it did not return to the levels seen in agematched healthy controls. In terms of the trophic (cytoarchitectural) changes and plastic (connectivity) changes in depressive disorder discussed here, the improvement in memory function observed by Abas and her colleagues following treatment for depression may represent the partial restoration of plastic capacities in the hippocampus; while the persisting decrement in performance in comparison with healthy controls might reflect the longer-term effects of cell loss.

Although there is little doubt that hippocampal damage has profound effects on cognitive function, it is unlikely that hippocampal dysfunction in depressive disorder is expressed purely as memory disorder. Rather than representing a passive casualty generating incidental memory failure, the dysfunctional hippocampus may also play an important role in the induction and maintenance of the depressed mood state itself. The hippocampus has a long and chequered 'career' in the neurosciences, and a variety of functions have been ascribed to the structure over the years. Early researchers considered the hippocampus to have a primarily olfactory function, but later conceptualisations include the hippocampus in limbic circuits believed to be concerned with the apprehension and expression of emotion. It now seems likely that the hippocampus contributes to a range of activities: although much contemporary research focuses on its role in learning and memory, the stress-related changes in hippocampal function reviewed above have led to a reappraisal of its functions, not least its role in the regulation of corticosteroid function.

\section{SYNTHESIS}

Drawing neurotrophic and plasticity hypotheses together, we suggest that stress may induce a continuum of effects, ranging from mild perturbation of plasticity with attendant cognitive and emotional dysfunction, to frank neural damage accompanied by more gross neuroanatomical changes - all orchestrated by the expression of BDNF. Individuals will occupy different positions on the continuum for any given stressor determined by genetic factors, early life experience, previous episodes of stress, cortisol response, prior neural damage, age, and so on. In this view, monoamine systems play a complementary, rather than central, role and represent but one potential mechanism by which depressive symptoms might ultimately be attenuated. This formulation perhaps goes some way towards explaining the perplexing variety of $\operatorname{cog}$ nitive, emotional, neuroanatomical and genetic findings (along with variations in 'treatability') encountered in depressive disorder. It may also help to understand the relationships between affective disorder and more obviously degenerative disorders, such as Alzheimer's disease or vascular dementia, where the more specific pathophysiological processes of the latter conditions assault a hippocampal system, already made vulnerable by psychological stressors.

We have tried to follow a continuous thread from molecular events in the nuclei of individual cells, through the dynamic properties of neural networks and the brain structures that they subserve, to neuropsychological phenomena and the effects of social stressors. While we recognise that the experimental findings presented here are derived almost exclusively from animal studies, and that a great deal is yet to be discovered, we believe that a 'competent' 
neurobiology of depressive disorder will eventually take this general form.

\section{ACKNOWLEDGEMENTS}

The authors' research described here was supported by the Wellcome Trust, the Sim Bequest of the Royal College of Physicians of Edinburgh, Wyeth UK and Organon Laboratories.

\section{REFERENCES}

Abas, M. A., Sahakian, B. J. \& Levy, R. (1990) Neuropsychological deficits and CTscan changes in elderly depressives. Psychological Medicine, 20, 507-520.

Altar, C. A. (1999) Neurotrophins and depression. Trends in Pharmacological Sciences, 20, 59-61.

Bazin, N., Perruchet, P., De Bonis, M., et al (1994)

The dissociation of explicit and implicit memory in depressed patients. Psychological Medicine, 24 239-245.

Drevets, W. C., Price, J. L., Simpson, J. R., et al (1997) Subgenual prefrontal cortex abnormalities in mood disorders. Nature, 386, 824-827.

Duman, R. S., Heninger, G. R. \& Nestler, E. J. (1997) A molecular and cellular theory of depression. Archives of General Psychiatry, 54, 597-606.

Gould, E., Tanapat, P., McEwen, B., et al (1998) Proliferation of granule cell precursors in the dentate gyrus of adult monkeys is diminished by stress. Proceedings of the National Academy of Sciences of the USA, 95, 3168-3171.

Henke, P. G. (1989) Synaptic efficacy in the entorhinaldentate pathway and stress ulcers in rats. Neuroscience Letters, 107, II0-113.

- (1990) Granule cell potentials in the dentate gyrus of the hippocampus: coping behaviour and stress ulcers in rats. Behavioural Brain Research, 36, 97-103.

Jeffery, K. \& Reid, I. C. (1997) Modifiable neuronal connections: an overview for psychiatrists. American Journal of Psychiatry, 154, 156-164.

Kehoe, P., Hoffman, J. H., Austin-LaFrance, R. J., et al (1995) Neonatal isolation enhances hippocampal dentate response to tetanization in freely moving juvenile male rats. Experimental Neurology, 136, 89-97.

Kim, J. J. \& Yoon, K. S. (1998) Stress: metaplastic effects in the hippocampus. Trends in Neurosciences, 2I, 505-509.

LeDoux, J. (1996) The Emotional Brain. New York: Simon and Schuster.

Levine, E. S., Crozier, R. A., Black, I. B., et al (1998) Brain-derived neurotrophic factor modulates hippocampal synaptic transmission by increasing $\mathrm{N}$-methyl-D-aspartic acid receptor activity. Proceedings of the National Academy of Sciences of the USA, 95 10235-10239.

Madsen, T. M., Treschow, A., Bengzon, J., et al (2000) Increased neurogenesis in a model of electroconvulsive therapy. Biological Psychiatry, 47, 1043-1049.

\section{CLINICAL IMPLICATIONS}

- New conceptualisation of the pathophysiology of depressive disorder are beginning to emerge.

- Novel therapeutic targets beyond the regulation of monoamine neuroreceptors are proposed.

- Chronic depression may represent a subtle neurodegenerative disorder: early and vigorous treatment of acute episodes may be important in the prevention of enduring cognitive dysfunction.

\section{LIMITATIONS}

Most, but not all, of the work described here is derived from non-human animal studies.

- Changes in neural connectivity cannot yet be studied directly in human subjects.

- More sophisticated models of the relationships between stress, limbic function and depressive disorder are required.

IAN C. REID, MRCPsych, CAROLINE A. STEWART, PhD, Department of Psychiatry, University of Dundee, Dundee

Correspondence: Professor lan C. Reid, Department of Psychiatry, University of Dundee, Ninewells Hospital, Dundee DDI 9SY

(First received 29 June 1999, final revision 30 July 2000, accepted 14 August 2000)

Pavlides, C., Kimura, A., Magarinos, A. M., et al (1995) Opposing roles of type I and type II adrenal steroid receptors in hippocampal long-term potentiation. Neuroscience, 68, 387-394.

\section{Petrie, R. X., Reid, I. C. \& Stewart, C. A. (2000)}

The NMDA receptor, synaptic plasticity and depressive disorder: a critical review. Pharmacology and Therapeutics, 87, II-25.

Reid, I. C. \& Stewart, C. A. (1997) Seizures, memory and synaptic plasticity. Seizure, 6, 35I-359.

Schaaf, M. J., de Jong, J., de Kloet, R., et al (1998) Downregulation of BDNF mRNA and protein in the rat hippocampus by corticosterone. Brain Research, 813, 112-120.

Shah, P. J., Ebmeier, K. P., Glabus, M. F., et al (1998) Cortical grey matter reductions associated with treatment-resistant chronic unipolar depression. Controlled magnetic resonance imaging study. British Journal of Psychiatry, 172, 527-532.

Siuciak, J. A., Lewis, D. R., Wiegand, S. J., et al (1997) Antidepressant-like effect of brain-derived neurotrophic factor (BDNF). Pharmacology, Biochemistry and Behavior 56, $131-137$

Skolnick, P. (1999) Antidepressants for the new millennium. European Journal of Pharmacology, 375, $31-40$.
Smith, M. A., Makino, S., Kvetnansky, R., et al (1995) Stress alters the expression of brain-derived neurotrophic factor and neurotrophin-3 mRNAs in the hippocampus. Journal of Neuroscience, I5, 1768-1777.

Stewart, C. \& Reid, I. (1993) Electroconvulsive stimulation and synaptic plasticity. Brain Research, 620 |39-141.

— \& (2000) Repeated ECS and fluoxetine administration have equivalent effects on hippocampal synaptic plasticity. Psychopharmacology, 48, 217-223.

Vaidya, V. A., Siuciak, J. A., Du, F., et al (1999) Hippocampal mossy fiber sprouting induced by chronic electroconvulsive seizures. Neuroscience, 89, 157-166.

Vickery, R., Morris, S. H. \& Bindman, L. J. (1997) Metabotropic glutamate receptors are involved in long-term potentiation in isolated slices of rat medial frontal cortex. Journal of Neurophysiology, 78, $3039-3046$

Xu, L., Holscher, C., Anwyl, R., et al (1998) Glucocorticoid receptor and protein/RNA synthesis dependent mechanisms underlie the control of synaptic plasticity by stress. Proceedings of the National Academy of Sciences of the USA, 95, 3204-3208. 